\title{
Primordial nucleosynthesis revisited via Trojan Horse Results
}

\author{
R.G. Pizzone ${ }^{1}$, R. Spartá ${ }^{1,2}$, C. Bertulani ${ }^{3}$, C. Spitaleri ${ }^{1,2}$, \\ M. La Cognata ${ }^{1}$, L. Lamia $^{2}$, A. Mukhamedzhanov $^{4}$ and \\ A. Tumino ${ }^{1,5}$
}

\author{
${ }^{1}$ INFN - Laboratori Nazionali del Sud, Catania, Italy \\ ${ }^{2}$ Dipartimento di Fisica e Astronomia, Università degli Studi di Catania, \\ Catania, Italy \\ ${ }^{3}$ Department of Physics and Astronomy, Texas A\&M University, \\ Commerce, TX 75025, USA \\ ${ }^{4}$ Cyclotron Institute, Texas A\& M University, College Station, TX, USA \\ ${ }^{5}$ Facoltà di Ingegneria e Architettura, Università Kore, Enna, Italy
}

\begin{abstract}
Big Bang Nucleosynthesis (BBN) requires several nuclear physics inputs and nuclear reaction rates. An up-to-date compilation of direct cross sections of $d(d, p) t, d(d, n){ }^{3} \mathrm{He}$ and ${ }^{3} \mathrm{He}(d, p){ }^{4} \mathrm{He}$ reactions is given, being these ones among the most uncertain bare-nucleus cross sections. An intense experimental effort has been carried on in the last decade to apply the Trojan Horse Method (THM) to study reactions of relevance for the $\mathrm{BBN}$ and measure their astrophysical $\mathrm{S}(\mathrm{E})$-factor. The reaction rates and the relative error for the four reactions of interest are then numerically calculated in the temperature ranges of relevance for $\mathrm{BBN}\left(0.01<\mathrm{T}_{9}<10\right)$. Their value were therefore used as input physics for primordial nucleosynthesis calculations in order to evaluate their impact on the calculated primordial abundances of $\mathrm{D},{ }^{3,4} \mathrm{He}$ and ${ }^{7} \mathrm{Li}$. These were compared with the observational primordial abundance estimates in different astrophysical sites. A comparison was also performed with calculations using other reaction rates compilations available in literature.
\end{abstract}




\section{Introduction}

One of the foundation stones of the Big Bang model, together with the Hubble expansion and the Cosmic Microwave Background (CMB) radiation [1] is the Big Bang Nucleosynthesis (BBN). BBN probes the Universe to the earliest times, the so called radiation dominated era, from a fraction of second to few minutes. It involves events that occurred at temperatures below $1 \mathrm{MeV}$, and naturally plays a key role in forging the connection between cosmology and nuclear physics [2]. Focusing only on the products of the BBN, according to the Standard Big Bang Nucleosynthesis model (SBBN), only the formation of light nuclei $\left({ }^{2} \mathrm{H},{ }^{3,4} \mathrm{He},{ }^{7} \mathrm{Li}\right)$ is predicted in observable quantities, starting from protons and neutrons. Today, with the only exception of ${ }^{3} \mathrm{He}$ and lithium, the abundances of these isotopes in the appropriate astrophysical environments are rather consistent with SBBN predictions [3]. A comparison between the primordial abundances from WMAP observations and the calculated ones constrains the baryon-to-photon ratio, $\eta$, which is the only free parameter in the presently accepted model of the SBBN. A recent observation yields $\eta=6.16 \pm 0.15 \times 10^{-10}$ [4], which is the value that we adopt in our calculations.

BBN nucleosynthesis requires several nuclear physics inputs and, among them, an important role is played by nuclear reaction rates. Due to the relatively small amount of key nuclear species involved in the BBN nuclear reaction network, only 12 reactions play a major role [5]. The reaction rates are calculated from the available low-energy cross sections for reactions which are also a fundamental information for a number of other still unsolved astrophysical problems, e.g. the so called "lithium depletion" either in the Sun or in other galactic stars $[6,7]$. Cross sections should be measured in the astrophysically relevant Gamow window [8], of the order of few hundreds $\mathrm{keV}$. In the last decades these reactions have been widely studied and, in particular, great efforts have been devoted to their study by means of direct measurements at the relevant astrophysical energies, sometimes in underground laboratories $[9,10]$. However, for many of the relevant reactions, no direct experiments exist at astrophysical energies (mostly because of difficulties connected with the presence of the Coulomb barrier in charged particle induced reactions) and the cross section within the Gamow window has to be extrapolated from higher energy measurements. Alternative and challenging ways to obtain $\sigma_{b}$ for charged-particles at sub-Coulomb energies have been provided by indirect methods such as the Coulomb dissociation method [11, 12] and the ANC (Asymptotic Normalization Coefficient) [13]. Among them, the Trojan-horse Method (THM) [14] is particularly suited 
to investigate binary reactions induced at astrophysical energies by neutrons or charged particles by using appropriate three-body reactions. It allows one to avoid both Coulomb barrier suppression and electron screening effects, thus preventing the use of unreliable extrapolations. In the next sections we will show the calculations of the reaction rates based also on the THM measurements of the cross sections $\sigma_{b}$. For recent reviews on the THM see $[15,16])$. Thus, the method can be regarded as a powerful indirect technique to get information on bare nucleus cross section for reactions of astrophysical interest, which leads to new reaction rates determination. Some of the reactions relevant for the SBBN, i.e. ${ }^{7} \mathrm{Li}(\mathrm{p}, \alpha){ }^{4} \mathrm{He},{ }^{2} \mathrm{H}(\mathrm{d}, \mathrm{p})^{3} \mathrm{H}$, ${ }^{2} \mathrm{H}(\mathrm{d}, \mathrm{n}){ }^{3} \mathrm{He},{ }^{3} \mathrm{He}(\mathrm{d}, \mathrm{p}){ }^{4} \mathrm{He}$, were studied by means of the THM in the energy range of interest and their measurements were performed in an experimental campaign which took place in the last decade [17-24].

\subsection{Reaction rates with TH data}

The reaction rates for the the four reactions mentioned above (from a compilation of direct and THM data, as reported in [25]), have been calculated numerically. Then, we fitted the rates with the parametrization displayed in Equation 1. This is the common procedure adopted in previous works (see, e.g., [26-28]). For the 4 reactions of interest, we have fully included the experimental errors from measurements, allowing us to evaluate the respective errors in the reaction rates. The numerical results are then fitted with the expression

$$
\begin{array}{r}
N_{A}\langle\sigma v\rangle=\exp \left[a_{1}+a_{2} \ln T_{9}+\frac{a_{3}}{T_{9}}+a_{4} T_{9}^{-1 / 3}+\right. \\
\left.a_{5} T_{9}^{1 / 3}+a_{6} T_{9}^{2 / 3}+a_{7} T_{9}+a_{8} T_{9}^{4 / 3}+a_{9} T_{9}^{5 / 3}\right]
\end{array}
$$

which incorporates the relevant temperature dependence of the reaction rates during the BBN. The $a_{i}$ coefficients for the ${ }^{2} \mathrm{H}(\mathrm{d}, \mathrm{p}){ }^{3} \mathrm{H},{ }^{2} \mathrm{H}(\mathrm{d}, \mathrm{n}){ }^{3} \mathrm{He}$, ${ }^{3} \mathrm{He}(\mathrm{d}, \mathrm{p}){ }^{4} \mathrm{He}$ and ${ }^{7} \mathrm{Li}(\mathrm{p}, \alpha){ }^{4} \mathrm{He}$ reaction rate expression are given in [25]. The direct data were considered from the compilation described in [25] for energies above $100 \mathrm{keV}$ for ${ }^{3} \mathrm{He}(\mathrm{d}, \mathrm{p}){ }^{4} \mathrm{He}$ and ${ }^{7} \mathrm{Li}(\mathrm{p}, \alpha){ }^{4} \mathrm{He}$ and for energies above $10 \mathrm{keV}$ for ${ }^{2} \mathrm{H}(\mathrm{d}, \mathrm{p})^{3} \mathrm{H}$ and ${ }^{2} \mathrm{H}(\mathrm{d}, \mathrm{n})^{3} \mathrm{He}$, in order to avoid the enhancement due to the electron screening in the direct data.

For all the cases we noticed that deviations of up to $20 \%$ are obtained from previous compilations. 
Table 1: BBN predictions using different set of data (see text) compared with observations. (a) The mass fraction for ${ }^{4} \mathrm{He}, Y_{p}$ is from Ref. [36]. (b) The mean deuterium abundance is the mean average from [37]. (c) The ${ }^{3} \mathrm{He}$ abundances are adopted from Ref. [38] as a lower limit to the primordial abundance. (d) The lithium abundance arises from observations of stars which provide a sample of the "lithium plateau" [39]. D/H is in units of $10^{-5},{ }^{3} \mathrm{He} / \mathrm{H}$ in $10^{-6}$ and $\mathrm{Li} / \mathrm{H}$ in $10^{-10}$.

\begin{tabular}{|l|c|c|}
\hline Yields & present work & Observed \\
\hline$Y_{p}$ & $0.2485_{-0.002}^{+0.001}$ & $0.256 \pm 0.006^{(a)}$ \\
\hline $\mathrm{D} / \mathrm{H}$ & $2.692_{-0.070}^{+0.177}$ & $2.82 \pm 0.26^{(b)}$ \\
\hline${ }^{3} \mathrm{He} / \mathrm{H}$ & $9.441_{-0.466}^{+0.511}$ & $\geq 11 . \pm 2 .^{(c)}$ \\
\hline${ }^{7} \mathrm{Li} / \mathrm{H}$ & $4.683_{-0.292}^{+0.335}$ & $1.58 \pm 0.31^{(d)}$ \\
\hline
\end{tabular}

\section{Discussion and conclusion}

The reaction rates of four of the main reactions of the $\mathrm{BBN}$ network in the temperature range $\left(0.001<\mathrm{T}_{9}<10\right)$, namely, ${ }^{2} \mathrm{H}(\mathrm{d}, \mathrm{p})^{3} \mathrm{H}, \mathrm{d}(\mathrm{d}, \mathrm{n})^{3} \mathrm{He}$, ${ }^{3} \mathrm{He}(\mathrm{d}, \mathrm{p}){ }^{4} \mathrm{He},{ }^{7} \mathrm{Li}(\mathrm{p}, \alpha){ }^{4} \mathrm{He}$, have been calculated numerically including the recent THM measurements [29-35]. The uncertainties of experimental data for direct and THM data have been fully included for the above reactions. The extension of the same methodology to the other reactions forming the $\mathrm{BBN}$ reaction network will be examined in a forthcoming paper. The parameters of each reaction rates as given in Eq. 1 are reported in [25]. The obtained reaction rates are compared with some of the most commonly used compilations found in the literature and used to calculate the BBN abundance for ${ }^{3,4} \mathrm{He}, \mathrm{D}$ and ${ }^{7} \mathrm{Li}$. The obtained abundances are in agreement, within the experimental errors, with those obtained using the compilation of direct reaction rates. Moreover, a comparison of our predictions with the observations for primordial abundance of ${ }^{3,4} \mathrm{He}, \mathrm{D}$ and ${ }^{7} \mathrm{Li}$ show an agreement for ${ }^{3,4} \mathrm{He}$ and $\mathrm{D}$, while showing a relevant discrepancy for ${ }^{7} \mathrm{Li}$ as reported in table 1 . The present results show the power of THM as a tool for exploring charged particle induced reactions at the energies typical of $\mathrm{BBN}$.

This work was supported by the Italian Ministry of the University under Grant No. RBFR082838 and by the Italian Ministry of University MIUR under the grant "LNS Astrofisica Nucleare (fondi premiali)". This work was partially supported by the US-DOE grants DE-FG02-08ER41533 and DE-FG02-10ER41706. A.M. acknowledges the support by US Department of Energy under Grant Nos. DE-FG02-93ER40773, DE-FG52- 09NA29467, and DE-SC0004958. 


\section{References}

[1] G. Steigman 2007, Ann. Rev. Nucl. Part. Sci. 57, 463

[2] B. D. Fields and S. Sarkar 2006, J. Phys. G33, 220

[3] G. Israelian 2012, Nature 489, 37

[4] E. Komatsu et al. 2011, Ap. J. Sup. 192, 18

[5] E.W. Kolb and M.S. Turner 1990, "The Early Universe", AddisonWesley

[6] R. Weymann and E. Moore 1963, Ap. J. 137, 552

[7] D. Ezer and A.G.W. Cameron 1963, Icarus 1, 422

[8] C. Iliadis 2007, "Nuclear Physics of Stars", Wiley

[9] R. Bonetti et al. 1999, Phys. Rev. Lett. 82, 5205

[10] C. Casella et al. 2002, Nucl. Phys. A706, 203

[11] G. Baur, C.A. Bertulani \& H Rebel 1986, Nucl. Phys. A458, 188

[12] C.A. Bertulani \& A. Gade 2010, Phys. Rep. 485, 195

[13] A. Mukhamezhanov et al. 2008, Phys. Rev. C 78, 0158042008

[14] C. Spitaleri et al. 2003, Nucl. Phys. A 719, 99c

[15] C. Spitaleri et al. 2011, Physics of At. Nucleus 74, 1725

[16] R.E. Tribble et al. 2014, Rep. Progr. Phys. 77, 10,106901

[17] A. Tumino et al. 2008, Phys. Rev. C 78, 064001

[18] R.G. Pizzone et al. 2005, A. \& A. 438, 779

[19] L. Lamia et al., 2013, Ap. J. 768, 65

[20] M. La Cognata et al. 2011, Ap. J. L. 739, L54

[21] L. Lamia, M. La Cognata, C. Spitaleri, B. Irgaziev, R.G. Pizzone 2012, Phys. Rev. C 85, 025805

[22] S. Romano et al. 2006, Eur. Phys. J. A 27, 221 
[23] Q. Wen et al. 2008, Phys. Rev. C 78, 035805

[24] S. Cherubini et al., 2015, Phys. Rev. C, 92, 015805

[25] R.G. Pizzone et al. 2014, Ap. J.,786, 112

[26] M.S. Smith, L.H. Kawano and R.A. Malaney 1993, Ap. J. 85, 219

[27] R.H. Cyburt 2004, Phys. Rev. D 70, 023505

[28] A. Coc, S. Goriely, Y. Xu, M. Saimpert, and E. Vangioni 2012, Astrophys. J. 744, 18

[29] L. Lamia et al. 2012, A. \& A. 541, 158

[30] M. Lattuada et al. 2001, Ap. J. 562, 1076

[31] A. Tumino et al. 2011, Phys. Lett. B 700 (2), 111

[32] A. Tumino et al. 2014, Ap. J., 785, 96

[33] M. La Cognata et al. 2005, Phys. Rev. C 72, 065802

[34] R.G. Pizzone et al. 2013, Phys. Rev. C 87, 025805

[35] Li C. et al., 2015, Phys. Rev. C, 92, 025805

[36] Y.I.Izotov and T.X.Thuan 2010, Ap. J. Lett. 710, L67

[37] J.M. O'Meara, S. Burles, J.X. Prochaska, and G.E. Prochter 2006, Ap. J. 649, L61

[38] T. M. Bania, R. T. Rood, D. S. Balser 2002, Nature 415, 54

[39] L. Sbordone, et al. 2010, Astron. Astro. 522, 26 